\title{
Síndrome de opsoclonos mioclonos causado por un neuroblastoma. Informe de un caso
}

\author{
Dr. Guillermo Dávila-Gutiérrez, * Dr. José Martín Palacios-Acosta, ${ }^{* *}$ Dra. Anabel Guzmán-Martínez, ${ }^{* * *}$ \\ Dr. Francisco Rodríguez-Abarca, ${ }^{* * * *}$ Dra. Jaime Shalkow-Klincovstein, ${ }^{1}$ Dr. Daniel Carrasco-Daza ${ }^{2 *}$
}

\section{RESUMEN}

Informamos el caso de una lactante mexicana con integridad neurológica previa, que desarrolló un síndrome de opsoclonos mioclonos (SOM), sin que tuviera algún proceso infeccioso intercurrente. Tuvo pobre respuesta al tratamiento con prednisona; mejoró parcialmente con inmunoglobulina intravenosa. Un gammagrama con metayodo-bencilguanidina mostró la presencia de tejido cromafín a nivel abdominal. Una tomografía computada de abdomen reveló un tumor abdominal. Los estudios de histopatología y extensión permitieron diagnosticar un neuroblastoma diferenciado en estadio 1. Se resecó el tumor y el paciente tuvo una evolución favorable.

Palabras clave: Síndrome de opsoclonos mioclonos, polimioclonos, ataxia, neuroblastoma, síndrome paraneoplásico.

\section{ABSTRACT}

We report herein the case of a 14 month-old Mexican female, without previous neurologic symptoms, who developed an opsoclonus myoclonus syndrome. There was no previous history of infectious processes. She responded poorly to prednisone and partially to intravenous immunoglobulin G. An MIBG scintigraphy showed chromaphin tissue in the abdomen. A CT-scan showed an abdominal mass. The tumor was completely resected, and a stage I differentiated neuroblastoma was confirmed by histopathology. Her neurologic status improved significantly after the surgical resection of the tumor.

Key words: Opsoclonus mioclonus syndrome, polimioclonus, ataxia, neuroblastoma, paraneoplastic syndrome.

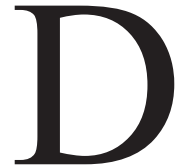
esde la primera descripción por Kaplan en $1959^{1}$, y la descripción clínica y precisa del síndrome de opsoclonos polimioclonos (SOM) por Kinsbourne ${ }^{2}$, se han informado aproximadamente 500 casos de SOM en la literatura mundial. Son

* Neurólogo pediatra adscrito al Servicio de Neurología

** Médico adscrito al Servicio de Cirugía Oncológica

*** Residente le la especialidad de Neurología Pediátrica

**** Residente de segundo año de Pediatría

Jefe del Servicio de Cirugía Oncológica

Patólogo pediatra adscrito al Servicio de Patología

Instituto Nacional de Pediatría

Los autores agradecen al Dr. Daniel Carrazco-Daza haber practicado el estudio histopatológico por el cual se llegó al diagnóstico definitivo.

Correspondencia: Dr. Guillermo Dávila-Gutiérrez. Instituto Nacional de Pediatría. Insurgentes Sur 3700-C. Col. Insurgentes Cuicuilco. México 04530 D.F. Tel: 10840900

Recibido: noviembre, 2009. Aceptado: diciembre, 2009.

Este artículo debe citarse: Dávila-Gutiérrez G, Palacios-Acosta JM, Guzmán-Martínez A y col. Síndrome de opsoclonos mioclonos causado por un neuroblastoma. Informe de un caso. Acta Pediatr Mex 2010;31(1):36-41.

La versión completa de este artículo también está disponible en: www.nietoeditores.com.mx escasos los informes en la población latinoamericana ${ }^{3-7}$. El SOM puede ser para o postinfeccioso ${ }^{6,8,9}$, paraneoplásico $^{3,10-13}$, asociado a procesos inmunopatológicos ${ }^{14} \mathrm{y}$ con frecuencia es criptogénico o incluso idiopático.

En países desarrollados, el síndrome paraneoplásico debido a tumores derivados de la cresta neural ocurre en 46 a $50 \%$ de los casos 3,12,15. En México está asociación es menos frecuente ${ }^{5,7,16}$. La tabla 1 muestra las posibles causas de SOM en niños y en adultos ${ }^{17-20}$.

El cuadro clínico del SOM se ve principalmente en lactantes. Se caracteriza por un inicio agudo, en ocasiones precedido o acompañado de un proceso infeccioso viral de las vías áreas superiores, gastroenteritis o exantema (Tabla 1). Súbitamente el paciente, de cualquier edad, desarrolla ataxia, temblores y polimioclonías, que lo incapacitan rápidamente para realizar la marcha independiente, la bipedestación, la sedestación, y tomar objetos con precisión, como llevarse los alimentos a la boca.

Los ojos se mueven rápidamente en todas direcciones en forma caótica pero conjugada (opsoclonos), que en ocasiones se asocia a movimientos palpebrales muy rápidos, llamado aleteo ("flutter"). Los pacientes se tornan 
Cuadro 1. Causas de SOM en niños y adultos

1. Idiopático o criptogénico: Probable síndrome paraneoplásico.

2. Paraneoplásico ( $50 \%$ de los casos en niños):

2.a. en población pediátrica: Tumores derivados de la cresta neural (neuroblastoma, ganglioneuroblastoma, ganglioneuroma). Hepatoblastoma.

2.b. en población adulta: Carcinoma de: pulmón, mama,riñón,vejiga. Linfoma melanoma, transplante alogénico de médula ósea.

3. Para o postinfeccioso: Virus del sarampión, parotiditis, rubéola, varicela, enterovirus, Epstein Barr, VIH, Salmonella $\mathrm{sp}$, Estreptococo piogenes, Rickettsias, Plasmodium sp, Borrelias, Mycobacterium tuberculosis.

4. Enfermedades inmunológicas: Síndrome de Elejalde, esclerosis múltiple.

5. Enfermedades degenerativas: Deficiencia de pantotenato cinasa, ataxia de Friedrich, enfermedad de Lafora.

6. Enfermedad vascular cerebral: Hemorragía talámica, insuficiencia basilar.

7. Otras: Hiperfosfatemia, diabetes mellitus tipo 1, enfermedad celíaca, hidrocefalia, coma hiperosmolar, displasias cerebrales, remedios herbales, fármacos como: diazepam, fenitoína, litio, amitriptilina, haloperidol, cocaína, y tóxicos: talio, tolueno, DDT.

muy irritables, llorones y en ocasiones agresivos. Pueden tener vómitos que causan deshidratación y desequilibrio hidroelectrolítico, alteraciones del tono muscular y de los reflejos de estiramiento. En la mayoría de los casos la evolución es crónica, con recaídas y periodos de mejoría que alternan sucesivamente ${ }^{2,5}$. Según Wilken y colaboradores, el $80 \%$ de los casos con SOM quedarán con secuelas neurológicas: déficit cognitivo, trastornos de conducta, trastornos de atención y de la sicomotricidad; pobre integración social y epilepsia ${ }^{21,22}$.

En 1864 Virchow describió el neuroblastoma ${ }^{23}$; en 1901 Beneke publicó el primer caso de ganglioneuroblastoma en un niño ${ }^{24}$; en 1928 Busch publicó el primer caso en un adulto ${ }^{25}$. En 1999, Shimada describió la clasificación patológica internacional para la evaluación pronóstica de los pacientes con tumores neuroblásticos; estableció subgrupos de pronóstico favorable o desfavorable ${ }^{26,27}$ :

- Neuroblastoma (pobre en estroma schwaniano).

- Ganglioneuroblastoma mixto (rico en estroma schwaniano).

- Ganglioneuroma (estroma schwaniano dominante).

- Ganglioneuroblastoma nodular.

Objetivo. Informar un caso de SOM debido a un neuroblastoma diferenciado en una lactante con el fin de familiarizar a los pediatras con el cuadro clínico y su posible etiología.

\section{CASO CLÍNICO}

Lactante femenina de 14 meses de edad, originaria de México, Distrito Federal, sin antecedentes de importancia ni cuadro infeccioso previo a su padecimiento. Cuenta con un esquema de inmunizaciones completo. Inició su padecimiento 14 días previos a su ingreso a nuestra Institución. En forma súbita y progresiva, presentó temblor de intención en las extremidades superiores y de la cabeza. Un pediatra le dio tratamiento con difenhidramina, sin que hubiera mejoría. Una semana después, el temblor se generalizó y aumentó de intensidad; el paciente se volvió irritable y le aparecieron movimientos oculares caóticos. Se hospitalizó en una institución particular donde recibió aciclovir, ácido valpróico, fenitoína, cefuroxime y nimesulida. Egresó de ese hospital con hipotonía generalizada. Una tomografía computada cerebral mostró dilatación del tercer ventrículo. Un día antes de su ingreso a nuestra Institución, su sintomatología aumentó y afectó gravemente todas sus funciones motoras, el lenguaje y mostró irritabilidad extrema.

Exploración física: Peso, $9 \mathrm{~kg}$; talla, $72 \mathrm{~cm}$; FC: 130 x', FR: 29 x', TA: 99/82 mm/Hg; PC, $46.5 \mathrm{~cm}$; temperatura, $36.1{ }^{\circ} \mathrm{C}$. Despierta, irritable, con periodos de somnolencia y ansiedad que mejoraban en el regazo de la madre y durante el sueño. Además presentaba llanto cefálico (irritabilidad extrema), ataxia troncal, dismetría, temblor de intención y opsoclonos que impedían la deambulación, la sedestación, tomar objetos con las manos, etc. Cráneo normal, pupilas isocóricas, con reflejos normales; fondo de ojo normal. Al inducir estrés y con los cambios de posición o la separación de su madre, aumentaba la sintomatología y se hacían más evidentes las polimioclonías generalizadas. El tono muscular era normal y la fuerza muscular era de 4/5; los reflejos de estiramiento eran normales y con respuesta plantar extensora bilateral. En ningún momento de su evolución hubo fiebre o signos meníngeos (Figura 1). Una tomografía computada del encéfalo mostró incremento en la dimensión del tercer ventrículo y de los ventrículos laterales, con discreta ampliación del espacio subaracnoideo.

Biometría hemática. $\mathrm{Hb}$, de $12.2 \mathrm{~g} / \mathrm{dL}$; leucocitos, 15000; neutrófilos, 38\%; linfocitos de 59\%, monocitos de $3 \%$, plaquetas de 244000 . Pruebas de coagulación, química sanguínea, electrolitos séricos y examen general de orina normales. Ultrasonido renal y de vías urinarias normales. Un electroencefalograma mostró ritmo lento generalizado de predominio en las regiones frontales y con 


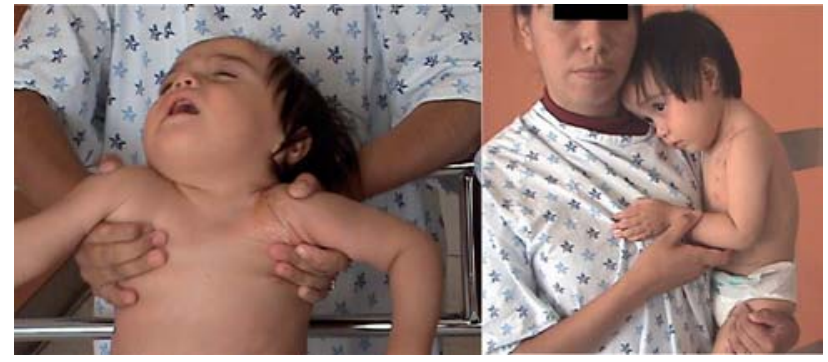

Figura 1. Paciente de 14 meses, con irritabilidad extrema, que se incrementa con los cambios de posición y disminuye al estar en contacto estrecho con la madre.

actividad paroxística de ondas agudas en ambas regiones frontales. Nunca tuvo crisis clínicas. Estudio citoquímico del líquido cefalorraquídeo traumático: sin película, rojo turbio; 500 eritrocitos.

Se le trató inicialmente con prednisona $2 \mathrm{mg} / \mathrm{kg} / \mathrm{día}$, ranitidina $3 \mathrm{mg} / \mathrm{kg} /$ día, clonazepam $0.4 \mathrm{mg}$ al día; no hubo respuesta adecuada. Al quinto día de su ingreso, un gammagrama con metayodo-bencilguanidina (Figura 2) mostró a nivel del abdomen en una proyección topográfica del riñón izquierdo, una zona positiva para tejido cromafín 28,29. Una tomografía computada de abdomen mostró una masa tumoral debajo de la bifurcación de la aorta, de $4 \mathrm{~cm}$ de diámetro (Figura 3). El estudio del frotis y la biopsia de médula ósea fueron negativos para células tumorales. Los anticuerpos séricos contra Epstein Baar, citomegalovirus, enterovirus, virus del sarampión y herpes resultaron negativos. Se le administró inmunoglobulina G (IgG) por vía intravenosa a dosis de $1 \mathrm{gm} / \mathrm{kg}$ y al séptimo día de $\mathrm{su}$ internamiento fue operada.

Se logró la resección completa del tumor sin complicaciones (Figura 4). El estudio histopatológico informó que el tumor y los ganglios regionales de la bifurcación de las arterias iliacas era un "neuroblastoma en diferenciación", pobre en estroma Schwaniano (menos del 50\%) que había roto la cápsula e infiltraba focalmente los tejidos blandos adyacentes. Dos ganglios linfáticos peritumorales regionales fueron positivos para infiltración tumoral. El índice de mitosis/cariorrexis era bajo, indicando un pronóstico favorable; existían calcificaciones distróficas focales ${ }^{30-34}$.

Un mes después de la cirugía disminuyeron las polimioclonías, el opsoclonos y la irritabilidad. Un estudio de seguimiento con metayodo-bencilguanidina fue negativo. La paciente sigue su tratamiento con prednisona $2 \mathrm{mg} / \mathrm{kg} /$ día.

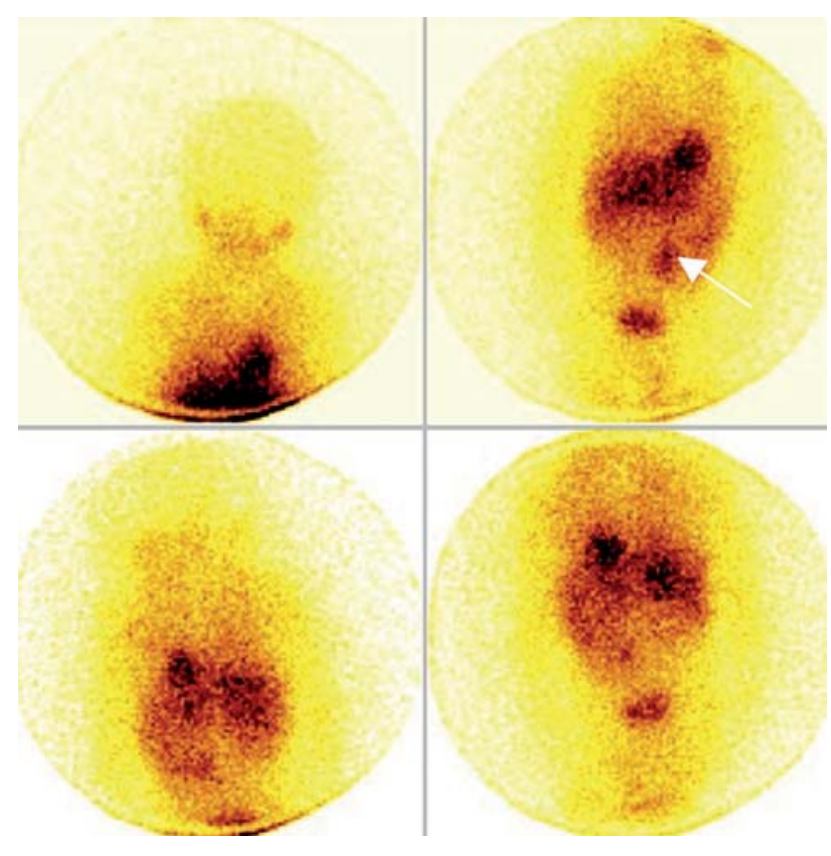

Figura 2. Estudio con MIBG demostrando el área positiva para tejido cromafín a nivel del abdomen (flecha).

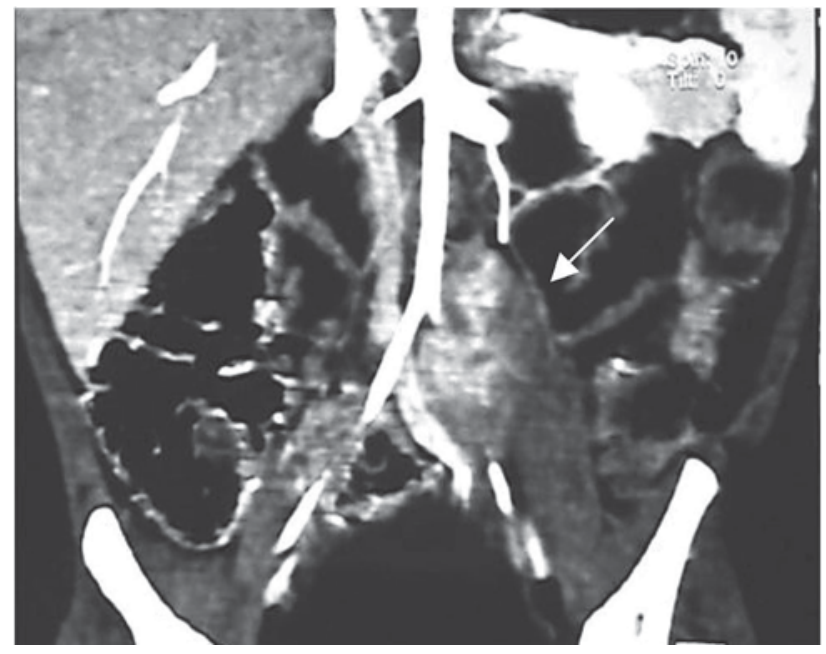

Figura 3. Tomografía computada de abdomen con medio de contraste, en corte coronal, que muestra la lesión tumoral bien circunscrita $(\rightarrow)$ y con calcificaciones, rodeando la porción proximal de la arteria iliaca común izquierda, que descansa sobre el músculo psoas ipsilateral y desplaza a la vena iliaca común hacia la línea media.

\section{Análisis}

El SOM es poco frecuente. Se desconocen su frecuencia y prevalencia en México. Su reconocimiento es clínico y exige un estudio urgente destinado a demostrar si existe o no un tumor oculto en el cuello, tórax, abdomen o en la 


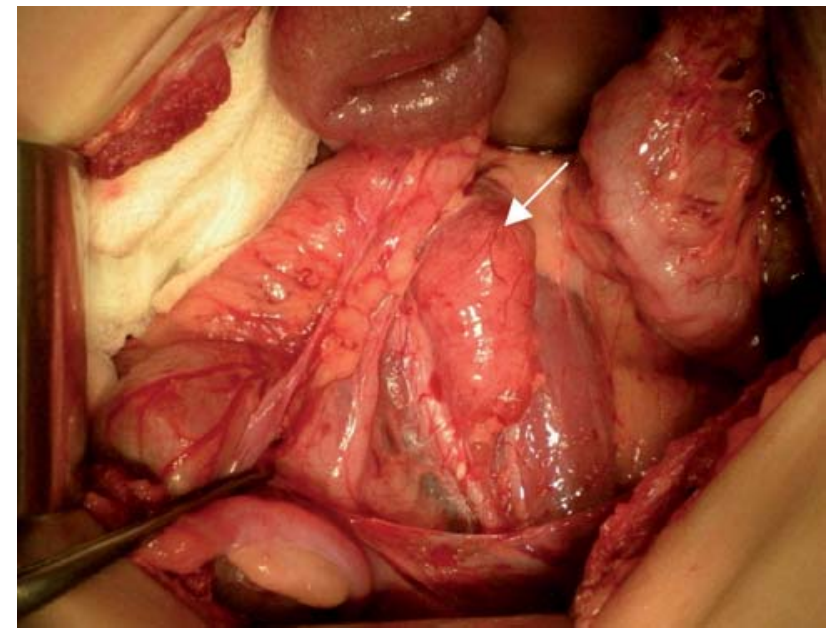

Figura 4. Imagen transoperatoria que muestra al tumor de origen neuronal sobre los vasos iliacos comunes del lado izquierdo $(\rightarrow)$; se encuentra debajo de la bifurcación de la aorta, entre el músculo psoas y la arteria iliaca común izquierda.

pelvis, ya que el SOM puede ser de carácter paraneoplásico causado por tumores derivados de la cresta neuronal o del hígado. El ganglioneuroblastoma mediastinal es el tumor que más frecuentemente se asocia con SOM; sin embargo, la asociación puede ocurrir con todos los tumores neuroblásticos de cualquier localización ${ }^{5,7,12,15,35}$.

En nuestra experiencia, el estudio más importante para demostrar el tejido cromafín oculto es la gammagrafía con metayodo-bencilguanidina complementado con una tomografía computada o con resonancia magnética cerebral. Por lo demás, la determinación de ácido vanillilmandélico, de catecolaminas en orina o de cistationina tienen menos sensibilidad y en ocasiones dan falsos negativos o falsos positivos ${ }^{28,29,35}$. Sin embargo, como sucedió en nuestra paciente, la hipertensión arterial era debida a la liberación de catecolaminas, lo cual, de sospecharse, hace conveniente cuantificarlas.

Descubierto el tumor, se procede a su estadificación para su tratamiento. La evolución después de su resección completa, es variable. Se han informado casos de remisión total de la sintomatología neurológica y casos con exacerbación de la misma. Por eso siempre es conveniente tomar en cuenta que el SOM puede estar presente varios meses antes de que aparezca cualquier evidencia bioquímica o por imagen del tumor ${ }^{36,37}$.

Cuando la sintomatología neurológica del paciente persiste, puede dejar secuelas, debido a lo cual se han pro- puesto varias alternativas terapéuticas. En los años ochenta y principios de los noventa del siglo pasado, existía ACTH en México a un costo accesible para la mayoría de los pacientes y la respuesta al tratamiento con frecuencia era adecuada. En la actualidad ya no se cuenta con ACTH y su costo en el extranjero es exorbitante lo cual ha hecho que se utilice IgG intravenosa asociada a esteroides y en los casos más refractarios, algunos autores recomiendan el uso de rituximab o de fármacos quimioterapéuticos ${ }^{38}$. En nuestra paciente estamos tratando (por falta de recursos económicos) de continuar el tratamiento con IgG.

La fisiopatología del SOM en pacientes con tumores derivados de la cresta neural se desconoce, pero todo indica que existe un proceso mediado por una respuesta inmunológica alterada que explica la buena respuesta a los esteroides, ACTH, plasmaféresis, IgG, rituximab y los fármacos quimioterapéuticos ${ }^{39-41}$. Se han identificado autoanticuerpos onconeuronales (anti-neuroblastoma) contra neuronas de Purkinje ${ }^{42}$. En adultos con SOM paraneoplásico se han detectado anticuerpos "Anti $\mathrm{Hu}$, Anti Ri y anti Yo", pero en la población pediátrica es raro encontrarlos $21,42,43$.

Prantazelli y cols. han demostrado que se puede reconocer el inmunofenotipo del SOM midiendo con citometría de flujo las subpoblaciones de linfocitos B (tipo CD 20,19,5), que están funcionalmente expandidos en el líquido cefalorraquídeo en pacientes con evolución desfavorable o pobre respuesta al tratamiento con esteroides, ACTH o IgG. Los linfocitos B pueden ser bloqueados y depletados con anticuerpos monoclonales "Rituximab" (anticuerpo monoclonal anti CD20) lo que mejora la sintomatología del SOM por periodos de seis a nueve meses ${ }^{38-46}$. En los casos graves y refractarios al tratamiento con esteroides e IgG se emplea el rituximab o fármacos como la ciclofosfamida, la azatioprina y el micofenolato de mofetilo ${ }^{21,47}$. Existen informes sobre el empleo de estos tratamientos a dosis variables con resultados también variables en pacientes con pobre respuesta al tratamiento habitual ${ }^{48-51}$.

\section{CONCLUSIONES}

El SOM es la forma severa de un espectro clínico que engloba a la ataxia aguda que evoluciona a la cronicidad, a la ataxia crónica con nistagmo o con opsoclonos y a la ataxia crónica con polimioclonías. Este espectro tiene una etiología variable que debe estudiarse con acuciosidad. 
Infortunadamente los fármacos útiles en los casos refractarios al tratamiento con esteroides son costosos. Por ello, es necesario buscar nuevas soluciones que ayuden a evitar las secuelas potenciales del SOM.

En todo niño o adolescente con SOM, el pediatra debe sospechar la posibilidad de que exista un síndrome paraneoplásico que debe ser estudiado y tratado rápidamente por un grupo interdisciplinario de especialistas. Es muy importante recordar que el niño con SOM se mueve para que lo vean, y mueve sus ojos en todas direcciones en forma caótica para sugerir que puede tener un tumor derivado de la cresta neural y se muestra irritable y con llanto cefálico pidiendo que lo lleven a un hospital de tercer nivel.

\section{BIBLIOGRAFÍA}

1. Kaplan M, Salet J, Pean G, et al. Sur une variété d'ataxie cérébelleuse acquise de l'enfance avec tremblement oculaire. Arch Fr Pédiatr. 1959;16: 1124-9.

2. Kinsbourne M. Myoclonic encephalopathy of infants. J Neurol Neurosurg Psychiatry. 1962;25: 271-9.

3. Brandt S, Carlsen N, Glenting P, Helweg-Larsen J. Encephalopathia myoclonica infantilis (Kinsbourne) and neuroblastoma in children. A report of three cases. Develop Med Child Neurol. 1974;16:286-94

4. Talon P, Stoll C. Syndrome opso-myoclonique de l'efant. Nouvelle observation. Revue de la literature (110 cas). Pediatrie. 1985;6:441-9.

5. Dávila-Gutiérrez G. Encefalopatía mioclonica infantil. Experiencia de 15 años en el diagnóstico y tratamiento. Tesis de Pediatría. Facultad de Medicina, División de Estudios Superiores de la UNAM. México, DF 1984.

6. Pranzatelli MR. The neurobiology of the opsoclonus- myoclonus syndrome. Clinical neuropharmacology. Clin Neuropharmacol. 1992;15:186-228.

7. Dávila-Gutiérrez G, García J. Experiencia de 27 años en el diagnóstico y tratamiento de la encefalopatía mioclónica infantil. Rev Mex Neuroci. 2009;10(2):151.

8. Kuban KC, EphrosMA, Freeman RL, Lafell LB, Bresan MJ. Opsoclonus myoclonus sindrome caused by Coxsackie B3 infection. Ann Neurol. 1983;13:69-71.

9. Ramos S, Temudo T. Síndrome de opsoclono-mioclono: ¿ ¿hasta cuándo seguir investigando? Rev Neurol. 2002;35(4):322-5.

10. Uroz TJ, Hernández C, Beltra R, Vidal R, Melwani M. Síndrome opsoclono-mioclono y neuroblastoma. Rev Oncol. 2004;6(3):170-2.

11. Ziegelbaum MM, Kay R, Rother D, Lorig R. The association of neuroblastoma with myoclonic encephalopathy of infants:the use of magnetic resonance as an imaging modality. J Urol. 1988;139:81-2.

12. Bukola BO, Shapiro S. Dancing eyes, dancing feet. Opsoclonus-myoclonus in an 18 month old child with neuroblastoma. Pediatr Emerg Care. 2007;23:885-8.

13. Aguilera S, Botella M, Salado C, Bosque A,Ocio I, Montiano
J. Síndrome opso-mioclono atáxico paraneoplásico. An Sist Sanit Navar. 2009;32:91-5.

14. Dávila GG. Síndrome de Elejalde en: Memorias de la Reunión Anual de la Academia Mexicana de Neurología. Chihuahua, México: 1988. p. 22.

15. Nickerson BG, Hutter J. Opsomyoclonus and neuroblastoma. Response to ACTH. Clin Pediatr. 1979;18.446-8.

16. Barragán PE, Velarde E, Garza M, Hernández A. Causas no oncológicas del síndrome de Kinsbourne. Bol Med Hosp Infant Mex. 2005;62:189-94.

17. Hunter S, Kooistra C. Neuropathologic findings in idiopathic opsoclonus and myoclonus. J Clin Neuro-ophthalmol. 1986;6:236-41.

18. Yakovlev PI. A case of myoclonus epilepsy with atrophy of brain-stem and Hallervorden Spatz type of pathologic change in the pallidum, substantia nigra and dentate nucleus. Trans Am Neurol Assoc. 1942;68:95-100.

19. Matsumma K, Sonoh M, Tomaoka A,Sakuta M. Syndrome of opsoclonus-myoclonus in hyperosmolar non ketotic coma. Ann Neurol. 1985;18:623-4.

20. Hoyt CM. Neonatal opsoclonus. J Pediatr Ophthalmol. 1977:14:274-7.

21. Wilken B, Baumann M, Bien C,Hero b, Rostasy K, Henefeld F. Chronic relapsing opsoclonus myoclonus syndrome: Combination of cyclophosphamide and dexamethasone pulses. J Eur Pediatrics Neurol. 2008;12:51-5.

22. Hayward K, Jeremy R, Jenkis S, Barkovich A, Gultekin S, Kramer J, Crittenden M, Matthay K. Long-term neurobehavioral outcomes in children with neuroblastoma and opsoclonus myoclonus ataxia syndrome: relationship to MRI findings and anti neuronal antibodies. J Pediatr. 2001;139:552-9.

23. Kelalis $P$, King L, Belman B. Adrenal, sympathetic chain and retroperitoneal tumors. In: Belman B, King L, Stephen A. Kramer S, eds. Clinical Pediatric Urology. 3rd ed. Philadelphia: WB Saunders; 2001. p. 1383-95.

24. Beneke R, von Zwei F. Ganglioneuroma. Beitr Pathol Anat. 1901;30:1-48.

25. Busch E. On ganglioneuroblastoma sympathicum. Pathol Microbiol Scand. 1928;5:289-305.

26. Shimada H, Umehara S, Monobe $Y$, Hachitanda $Y$, Nakagawa A, Goto S, et al. International neuroblastoma pathology classification for prognostic evaluation of patients with peripheral neuroblastic tumors. Cancer. 2001;92:2451-61.

27. Peuchmaur M, D’Amore E, Joshi V, Hata J, Borghild R, Dehner LP, et al.Revision of the international neuroblastoma pathology classification. Confirmation of favorable and unfavorable prognostic subsets in ganglioneuroblastoma nodular. Cancer. 2003;98:2274-81.

28. Shapiro B. Locating neuroblastoma in the opsoclonus-myoclono syndrome. J Nucl Biol Med. 1994;38:545-55.

29. Aldrich PM, Shalaby-Rana E,Movassaghi N,Majd M. The role of 131 iodine metaiodobenzylguanidine scanning in the correlative imaging of patients with neuroblastoma. Pediatrics. 1996;97:246- 50.

30. Stefanowicz J, Izycka-Swieszewska E, Drozynska E, Pienczk J, Polczynska K, Czauderna P. Neuroblastoma and opsoclonus-myoclonus-ataxia syndrome-clinical and pathological characteristics. Folia Neuropathol. 2008;46(3):176-85.

31. Raffaghello L,Conte M, De Grandis E, Pistoia V. Immunological mechanisms in opsoclonus-myoclonus associated neuroblas- 
toma. Eur J Paediatr Neurol. 2009;13:219-23.

32. Martin A, Messineo A,Lionetti P,Volta U, Stanghellini V, Barbara $G$. A case of paraneoplastic inflammatory neuropathy of the gastrointestinal tract related to an underlying neuroblastoma: successful management with immunosuppressive therapy. J Pediatr Gastroenterol Nutr. 2008;46:457-60.

33. Klein A, Schmitt B, Boltshauser E. Long-term outcome of ten children with opsoclonus-myoclonus syndrome. Eur J Pediatr. 2007;166:359-63.

34. Drukker CA,Heij H, Wijnaendts L, Verbeke J, Kaspers G. Paraneoplastic gastrointestinal anti Hu syndrome in neuroblastoma. Pediatr Blood Cancer. 2009;52:396-8.

35. Artigas PE, Sánchez E, Marlon V, Dávila G. Tumores derivados de la cresta neural en pacientes con encefalopatía opsomioclónica (Síndrome de Kinsbourne). Experiencia de 20 años con niños mexicanos. Acta Pediatr Mex. 2006;27(4):245-6.

36. Wheeler DS, Starr S. Case 2 presentation. Opsoclonusmyoclonus syndrome. Pediatr Rev. 1998;19:281-3.

37. Phol KRE, Pritchard J, Wilson J. Neurological sequelae of the dancing eye syndrome. Eur J Pediatr. 1996;155:237-44.

38. Pranzatelli RM, Tate E, Travelstead A, Longge D. Immunologic and clinical responses to Rituximab in a child with opsoclonusmyoclonus syndrome. Pediatrics. 2005;115:115-9.

39. Yiu VWY, Kovithavongs T, Mc Gonigle L, Ferreira P. Plasmapheresis as an effective treatment for opsoclonus myoclonus syndrome. Pediatr Neurol. 2001;24:72-4

40. Armstrong BM, Robertson L, Castle P. Delay, recurrent opsoclonus-myoclonus syndrome responding to plasmapheresis. Pediatr Neurol. 2005;33:365-7.

41. Veneselli E, Conte M, Biancheri R, Acquaviva A, De Bernardi B. Effect of steroid and high dose immunoglobulin therapy opsoclonus-myoclonus syndrome occurring in neuroblastoma. Med Pediatr Oncol. 1998;30:15-7.

42. Conolly AM, Pestronk A, Metha S, Prantazelli M, Noetzel M. Serum antibodies in childhood opsoclonus-myoclonus syn- drome: an analisis of antigenic targets in neuronal tissues. $\mathrm{J}$ Pediatr. 1997;130:878-84.

43. Fisher PG, Wechsler D, Singer H. Anti Hu antibody in neuroblastoma associated paraneoplastic syndrome. Pediatr Neurol. 1994;10:309-12

44. Corapcioglu F, Mutlu H, Kara B, Inan N, Akansel G,Gürbüz $Y$. Response to rituximab and prednisolone for opsoclonusmyoclonus-ataxia syndrome in a child with ganglioneuroblastoma. Pediatr Hematol Oncol. 2008;25:756-61.

45. Pranzatelli RM, Tate E, Travelstead A, Barbosa J, Bergamini $A$, Civitello $L$, et al. Rituximab (anti CD20) adjunctive therapy for opsoclonus- myoclonus syndrome. Pediatrics. 2006;28:58593.

46. Pranzatelli RM, Tate E, Hoefgen E, Wan J, Colliver J. Therapeutic down-regulation of central and peripheral B-cell activating factor (BAFF) production in pediatric opsoclonus myoclonus syndrome. Cytokine. 2008;4:26- 32.

47. Pranzatelli RM, Tate E, Travelstead L,Baumgarder A, Gorda $\mathrm{V}$, Haltore $\mathrm{N}$ y cols. Insights on chronic-relapsing opsoclonusmyoclonus from pilot study of mycophenolate mofetil. J Child Neurol. 2009;24:316-21

48. Chang HB, Koch T, Hopkins K, Malempati S. Neuroblastoma found in a 4 year old after rituximab therapy for opsoclonusmyoclonus. Pediatr Neurol. 2006;35:213-5.

49. Pranzatelli RM, Tate E, Travelstead L, Crowley, Toennies B, Creer M. Neurometabolic effects of ACTH on free amino compounds in opsoclonus- myoclonus syndrome. Neuropediatrics. 2008;39:164-71.

50. Bell J, Moran C, Blatt J. Reponse to rituximab in a child with neuroblastoma and opsoclonus- myoclonus. Pediatr Blood Cancer. 2008;50:370-1.

51. Leen GW, Weemaes C, Verbeek M, Willemsen M, Rotteveel J. Rituximab and intravenous immunoglobulins for relapsing postinfectious opsoclonus myoclonus. Pediatr Neurol. 2008;39: 213-7. 